\title{
Safe Eating of Fermented Corn and Coconut Food: Mechanism, Clinical Manifestations and Inhibition of Food Poisoning Involved in Bongkrekic Acid
}

\author{
Qingyu Huang ${ }^{1, *, \dagger}$, and Zhentian $\mathrm{Wu}^{2, \dagger}$ \\ ${ }^{1}$ Franklin and Marshall College, Lancaster, Pennsylvania 17603, US. \\ ${ }^{2}$ Suzhou Foreign Language School, Suzhou, Jiangsu, 215011, China. \\ †These authors contribute equally.
}

\begin{abstract}
Bongkrekic acid (BA) is a colorless and tasteless toxic. It exists in some fermented food and can be easily taken in by accident. Currently, no proper treatment has not been developed to treat this toxin in human body, therefore the death rate is very high. Mechanism of BA has been well studied these years and found that BA inhibit ATP synthesis, which lead to severe clinical symptoms, such as limb soreness, weakness and liver damage. In this paper, we mainly focus on the mechanism of BA in our human body, its related cases and the inhibition of BA in the food. This review can provide basic information for the future development of the treatment of BA in human body.
\end{abstract}

\section{Introduction}

Bongkrekic acid (BA) is a sort of fatty acid produced by the bacterium Burkholderia gladioli pv. Cocovenenans, which survives in corn flour and coconut products (Travis M Falconer et al., 2016). First documented food poisoning case due to BA happened in Indonesia in 1895. The causes of this bongkrekic poisoning were the consumption of tempeh, a famous Indonesia food made by coconut (William Shurtleff and Akiko Aoyagi, 2007). BA is a sort of mitochondrial toxins that damage mitochondria's function by inhibiting the activity of the (Adenosine triphosphate)ATP/(adenosine diphosphate)ADP carrier (Jonathan J. Ruprecht, et al., 2019), which causes the deficiency of energy used by cells in term of ATP.

Therefore, this foodborne illness related to BA lead to multi-aspect symptoms. At present, the treatment is immature (Travis M Falconer et al., 2016). It is best for people to understand how BA works as a mitochondrial toxin, why consuming fermented corn and coconut products causes BA related food poisoning, what serious symptoms are and how to inhibit BA. This paper will focus on the mechanism of BA, the relationship between $\mathrm{BA}$ and fermented corn food, clinical manifestations and the inhibition of BA.

\section{Mechanism}

It was generally known that $\mathrm{BA}$ inhibits mitochondrial enzymes. In the earliest studies, Welling investigated the cellular pathophysiology of BA, they showed dosedependent decreases in glucose content and cellular oxygen uptake in sheep heart tissue, along with lactate accumulation and acidosis. This illustration of BA site of action shows the inhibition of ATP translocase in the mitochondria matrix membrane, (as shown in the Figure 1), the cytoplasmic side of the carriernis closed by conserved hydrophobic residues. Glycine and small amino acid residues allow close-packing of helices on the matrix for ATP synthesis.

Solute transport across the impermeable mitochondria inner is essential for the function of survival of eukaryotic cells. This diffusion is catalyzed by membrane proteins. However, the carrier cycles between the cytoplasmic open state(C-state), and the matrix-open state (m-state 0$)$. BA inhibits the carrier by binding deeply and asymmetrically with in the central cavity. As a result, the carrier cannot switch between the two states, which inhibits the transport of materials on both sides of the membrane. In a word, BA interferes with the transport process of ADP and hinders the exchange of ADP and ATP between the mitochondrial inner membrane to produces toxic effects,-which directly affect the energy supply of biological cells.

\footnotetext{
*Corresponding author: qhuang@fandm.edu
} 


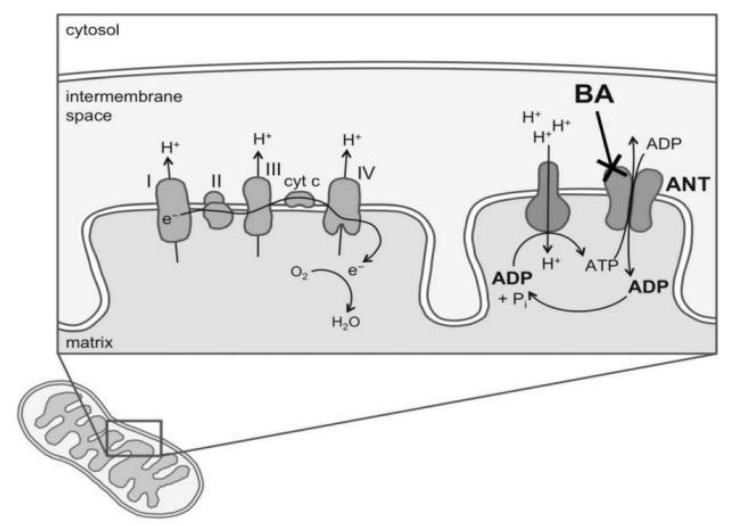

Figure 1. Inhibition of adenine nucleotide translocase in the mitochondrial matrix membrane by bongkrekic acid. (Mehruba Anwar et al., 2017)

\section{Bongkrekic acid and fermented corn food}

\subsection{Overview of Bongkrekic acid}

The molecular formula of $\mathrm{BA}$ is $\mathrm{C}_{28} \mathrm{H}_{38} \mathrm{O}_{7}$. Chemical structure of BA is shown in Figure 2. BA is produced by B. cocovenenans in warm environments $\left(22-30{ }^{\circ} \mathrm{C}\right)$ with a neutral $\mathrm{pH}$ value. The physical properties of $\mathrm{BA}$ are odorless and tasteless as well as heat-resistant, which reveals the reason why people will get food poisoning after consuming fermented corn and coconut food.

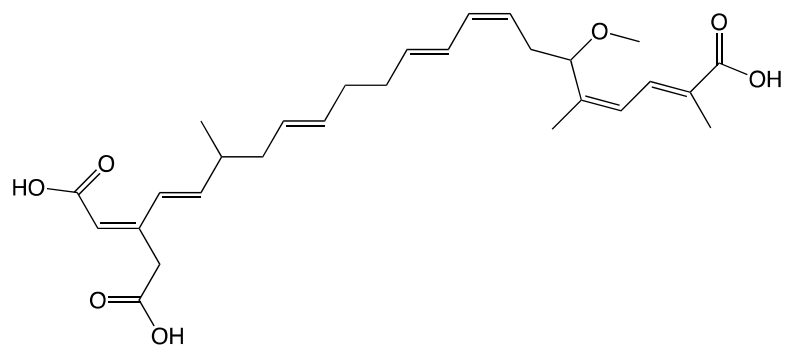

Figure 2. Chemical structure of Bongkrekic acid $\mathrm{C}_{28} \mathrm{H}_{38} \mathrm{O}_{7}$

\subsection{Relationship between Bongkrekic acid and fermented corn food}

From 1951 to 2014, up to 9336 people in Indonesia got BA poisoning by eating corn and coconut food, and 1112 people dead (Mehruba Anwar et al., 2017). Around 2359 people got BA poisoning and 905 people dead in China as shown in Figure 3(Mehruba Anwar et al., 2017). According to the records, Indonesia and China are two high incidence countries with BA poisoning every year. Morbidity and mortality present a strong correlation between BA and fermented corn food. To prove it, scientists did experiments with mice to prove that BA is the toxin in corn food that causes food poisoning. One group of mice were fed with pure toxin: BA, while another group of mice was fed with food made of fermented corn. The result of this experiment showed that two groups of mice have the same symptoms, which are respiratory failure and paralysis of the extremities $(\mathrm{Hu}$ wenjuan and Chen Xiaoming et al., 1989) Hence, it was shown that two groups of mice had the same food-borne illness because of BA.

This paragraph will examine the relationship between the production of BA and the different stages of the fermented corn food production process. In the first stage, corn and coconut is the raw material of fermented corn food. There exists oleic acid, a sort of fatty acid, in corn and coconut. Oleic acid provides B. cocovenenans with a suitable condition to live and produce BA (Mehruba Anwar et al. 2017). Hence, raw materials people used to produce food may contain BA. In the food production stage, the fermentation of several corn and coconut food like tempe, a type of traditional African foods made from fermented coconut pulp, has similar BA production conditions (J.M. Cox et al., 2014). The last stage is the cooking of corn and coconut food. The physical property of BA is odorless and tasteless, which is the reason why consumers can't realize the existence of BA in their food (Mehruba Anwar et al. 2017). Moreover, recent research revealed the heat-resistance property of BA (J.M. Cox et al., 2014). Therefore, BA remains in food and causes food poisoning even after cooking at a high temperature. Those two physical properties of BA can explain the reason why there are lots of victims who got alimentary toxicosis and even dead from eating fermented corn and coconut food.

In conclusion, the raw material used to produce food is easily "polluted by B. cocovenenans". Later, BA still can survive and doesn't be found in the process of food production and cooking because of its odorless, tasteless, and heat-resistant properties. Therefore, the final fermented corn food consumed still contains BA, which is the toxin that causes the food poisoning.

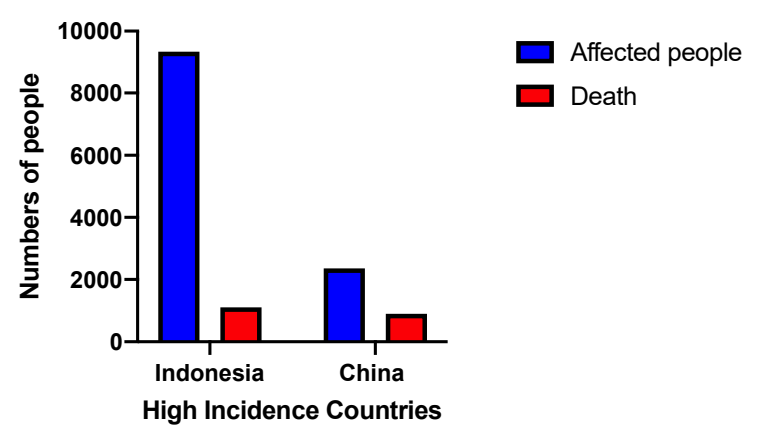

Figure 3. Recorded numbers of affected people and death in bongkrekic acid food poisoning (Mehruba Anwar et al., 2017)

\section{Clinical manifestations}

\subsection{Limb soreness and weakness}

Limb soreness and weakness are the common symptoms that exists in the outbreaks of the food-borne illness involved in BA. In the experiment, the mice had paralysis of the extremities before death (Hu wenjuan and Chen Xiaoming et al., 1989). According to the records, doctors found similar symptom existence in the patients with BA related food poisoning cases. For example, in the first documented BA poisoning case in Africa, 12 out of 17 interviewed survival in the case reported the limb weakness symptom after consuming pombe (Eduardo Samo Gudo,2018). In China, a 34-year-old male felt limb 
soreness and weakness after eating expired noodles in a noodles shop, which is got food poisoning due to BA.

With the increase of BA level in human body, glycogen metabolism is interfered by BA. As body regulation, liver and muscle glycogen are utilized by the body, which causes the decrease in intracellular glucose and oxygen consumption in the process. This process causes the accumulation of lactic acid produced in the decomposition of muscle glycogen. And the accumulation of lactic acid is the reason why patients feel muscle soreness and weakness (Ruijuan Shi and Chaoyang Long et al., 2019). Hence, if people feel muscle soreness and weakness after consuming fermented corn food, they shouldn't ignore the symptom and go to the hospital to examine if they got food poisoning due to BA.

\subsection{Liver damage}

Besides, there are many more clinical manifestations like digestive dysfunction, liver damage, and so on. Liver damage is one of the most severe symptoms. The examination of a 34-year-old male who got BA poisoning showed that he suffered from fatty liver, which indicated that he had fat metabolism disorder, and serious hepatic encephalopathy. Another 18-year-old woman in China also got fatty liver after eating rice noodles. After consuming expired corn-made- noodle, CT of a 23-yearold man shows the fatty liver (Ruijuan Shi and Chaoyang Long et al., 2019). On 23rd October 2018, seven people (including grandparents, parents (around 25 years old), a 3 -year-old son, a 2 years 5 months daughter, and 1 year old baby.) from one family and their dog got BA poisoning and had liver damaged after eating rice noodles (Jinghua li and et al., 2019). Liver damage is not a rare clinical manifestation, but rather an extremely common clinical manifestation on different-age-level human victims and animals who had food poisoning related to BA.

Some scientists conducted the experiment with rats to explain the reason why there are lots of pathological changes in liver. Scientists injected BA with tritium markers into the rats, which allows them to trace the flow of BA. After 15 minutes, most BA can be detected in the liver. Later, the experimental results presented that 70 percent of BA was excreted into feces and urine, while 30 percent of it was found in bile. After 6 hours, scientists could trace the metabolites of BA in the plasma and intestinal tract, which means that the metabolites of BA were transported from the bile to the intestinal tract. However, in the process of enterohepatic circulation, some metabolites of BA were reabsorbed by the liver. Therefore, this experiment concludes that the liver is damaged twice in the whole process. Therefore, liver damage is much more common and severe in this food positioning illness (Ruijuan Shi and Chaoyang Long et al., 2019).

In the future, it is worth for people to investigate drugs that can help to increase the excretion of BA from the body. Also, scientists can research how to stop the metabolites of BA from going to the enterohepatic circulation to avoid second entry of it into the liver. These are two directions to develop treatments for BA food poisoning.

\section{Inhibition of the production of Bongkrekic Acid in food}

Currently, only a few researches are done in terms of inhibiting BA in human body. One research paper states that loads of genetic tools previously developed for other Gram-negative bacteria are changed for use in BGC (Somprasong. Net al., 2010). Still, there is no clear inhibition method for human body. Thus, we turn our focus on inhibition the production of BA in food.

\subsection{The effect of salt and $\mathrm{pH}$ value on Bongkrekic acid production}

BA can be effectively inhibited with the existence of some salt and certain $\mathrm{pH}$ value. Buckle and his colleague examined the inhibition of BA in a traditional Indonesian food--Tempe bongkrek(Buckle, K. and Kartadarma, E., 1990). The food can be contaminated by Burkholderia gladioli pathovar cocovenenans (B. cocovenenans) which can produce at least two poisonous substances, bongkrek acid(BA) and toxoflaxin(TF). They use 3 different cocovenenanses which are all likely to exist when making the food. And different conditions are adjusted by controlling either the $\mathrm{pH}$ value or the amount of salt. The result shows that the production of $\mathrm{BA}$ and $\mathrm{TF}$ in either coconut culture medium(CCM) or tempe bongkrek is inhibited for all strains of B.cocovenanuns tested when the medium contained $2 \%$ salt and the $\mathrm{pH}$ is adjusted to 4.5 with acetic acid. (Figure 4 and Figure 5)

\subsection{The effect of lipids on Bongkrekic acid production}

The concentration and type of lipid in the substrate are critical for BA formation. The effect of lipid concentration and fatty acid type on the production of BA by $\mathrm{B}$. cocovenenans was examined by adding different amounts of coconut fat or individual free fatty acids to defatted and sterilized Rich Coconut Media $(\mathrm{dRCM})($ Garcia, R.A. et al., 1999) 'Forty and 50\% coconut fat resulted in as much as $1.4 \mathrm{mg} / \mathrm{g}$ BA (dry weight) at the same level of growth. Of eight saturated fatty acids tested, only lauric (12:0), myristic (14:0), and palmitic (16:0) acids stimulated the production of detectable amounts of toxin. When four 18-carbon free fatty acids with different degrees of saturation were compared, significant amounts of BA $(2.62 \mathrm{mg} / \mathrm{g}$ dry weight) were produced only with oleic acid (18:1).' The data above shows different types of lipid can make a huge difference on BA production. Therefore, with specific lipid type and concentration, BA can be inhibited effectively. 


\section{Toxin inhibition in tempe bongkrek}

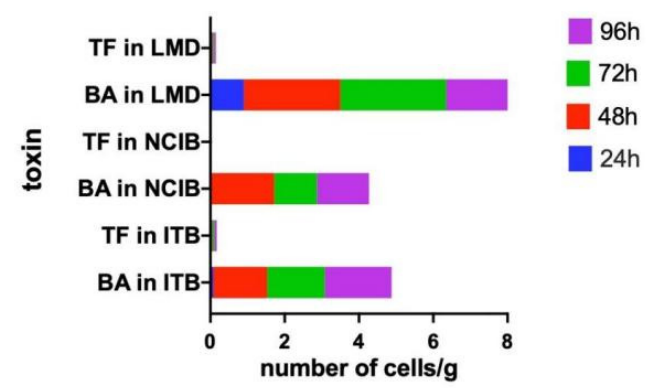

Figure 4. Mean toxin production (mg/g) by Pseudomonas cocovenenans strain ITB, NCIB and LMD in coconut culture medium during incubation at $30^{\circ} \mathrm{C}$

Different conditions for the toxin production

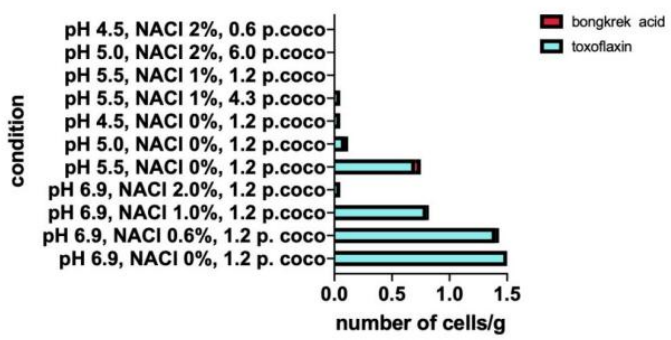

Figure 5. Bongkrek acid and toxoflavin Production $(\mathrm{mg} / \mathrm{g})$ by Pseudomonas cocovenenans strain ITB after $48 \mathrm{~h}$ incubation at $30^{\circ} \mathrm{C}$ in coconut culture medium treated with sodium chloride and acetic acid

\section{Conclusion}

BA, as a colorless and tasteless toxic, has caused high mortality rate in some Asia areas. And people do not have a proper treatment for it. Up till now, one of the most efficient ways for us to take control of the high mortality rate is to inhibit the production of BA. Teaching people how to take care of specific food by controlling the salinity and $\mathrm{pH}$ value and going hospital when performing some symptoms are crucial to avoid illness and death.

As we have introduced the mechanism of BA, BA and fermented corn food, clinical manifestations and the ways to inhibit the production of BA, we can know how BA works in our body, how people will react after get this poison, directions for treating this toxin and how to inhibit this toxin in food production. These are all very helpful for us to understand BA thoroughly. However, the proper treatment in human body is still unknown. Hopefully, as we learn more about this toxin, the high fatality rates caused by this toxin can decrease.

\section{Reference:}

1. M. Anwar, A. Kasper et al. Bongkrekic Acid-a Review of a Lesser-Known Mitochondrial Toxin. J. Med. Toxicol. 173-179 (2017)

2. Hu wenjuan and Chen Xiaoming et al. Fermented corn flour poisoning in rural areas of China III. Isolation and Identification of main toxin produced by causal microorganisms. Biomedical and environmental science 2, 65-71 (1989)

3. Ruijuan Shi and Chaoyang Long et al. Bongkrekic acid poisoning: Severe liver function damage combined with multiple organ failure caused by eating spoiled food. Legal Medicine 41, 2-6 (2019)

4. J. J. Ruprecht and M. S. King et al. The Molecular Mechanism of Transport by the Mitochondrial ADP/ATP Carrier. Cellpress 435 (2019)

5. Falconer, T. M., Kern, S. E., Brzezinski, J. L., Turner, J. A., Boyd, B. L., \& Litzau, J. J. Identification of the potent toxin bongkrekic acid in a traditional African beverage linked to a fatal outbreak. Forensic science international, 270, e5-e11.(2017) https://doi.org/10.1016/j.forsciint.2016.10.015

6. J.M. Cox, K.A. Buckle, E. Kartadarma PSEUDOMONAS | Burkholderia gladioli pathovar cocovenenans, Editor(s): Carl A. Batt, Mary Lou Tortorello, Encyclopedia of Food Microbiology (Second Edition), Academic Press, 248-252 (2014)

7. Li, J., Zhou, L. U., Long, C., Fang, L., Chen, Q., Chen, Q., Liang, J., Yang, Y., Zhu, H., Chen, Z., Gao, S., Li, Z., Li, Q., Huang, Q., \& Zhang, Y. An Investigation of Bongkrekic Acid Poisoning Caused by Consumption of a Nonfermented Rice Noodle Product without Noticeable Signs of Spoilage. Journal of food protection, 82(10), 1650 1654. (2019) https://doi.org/10.4315/0362-028X.JFP-19-121

8. Somprasong, Mcmillan, Karkhoff-Schweizer, Mongkolsuk, \& Schweizer. Methods for genetic manipulation of Burkholderia gladioli pathovar cocovenenans. BMC Research Notes, 3, 308 - 308. (2010)

9. Buckle, K., \& Kartadarma, E. Inhibition of bongkrek acid and toxoflavin production in tempe bongkrek containing Pseudomonas cocovenenans. The Journal of applied bacteriology, 68 6, 571-6 . (1990)

10. Garcia, R.A., Hotchkiss, J., \& Steinkraus, K. The effect of lipids on bongkrekic (Bongkrek) acid toxin production by Burkholderia cocovenenans in coconut media. Food additives and contaminants, 16 2, 63-9. (1999)

11. Gudo, E. S., Cook, K., Kasper, A. M., Vergara, A., Salomão, C., Oliveira, F., Ismael, H., Saeze, C., Mosse, C., Fernandes, Q., Viegas, S. O., Baltazar, C. S., Doyle, T. J., Yard, E., Steck, A., Serret, M., Falconer, T. M., Kern, S. E., Brzezinski, J. L., Turner, J. A., ... Chitima Investigation Group. Description of a Mass Poisoning in a Rural District in Mozambique: The First Documented Bongkrekic Acid Poisoning in Africa. Clinical infectious diseases : an official publication of the Infectious Diseases Society of America, 66(9), 1400-1406. (2018) https://doi.org/10.1093/cid/cix1005

12. William Shurtleff and Akiko Aoyag. History of Tempeh, History of Soybeans and Soyfoods: 1100 B.C. to the 1980s, 13 (Soyinfo Center, Lafayette, California, 2007) 